\title{
Travelling-waves consistent with turbulence-driven secondary flow in a square duct
}

\author{
Markus Uhlmann ${ }^{1}$, Genta Kawahara ${ }^{2}$ and Alfredo Pinelli ${ }^{3}$ \\ ${ }^{1}$ Institute for Hydromechanics, Karlsruhe Institute of Technology, 76128 Karlsruhe, Germany \\ ${ }^{2}$ Department of Mechanical Science, Osaka University, 560-8531 Osaka, Japan \\ ${ }^{3}$ Modeling and Numerical Simulation Unit, CIEMAT, 28040 Madrid, Spain
}

(Dated: 3 November 2010)

We present numerically determined travelling-wave solutions for pressure-driven flow through a straight duct with a square cross-section. This family of solutions represents typical coherent structures (a staggered array of counter-rotating streamwise vortices and an associated low-speed streak) on each wall. Their streamwise average flow in the cross-sectional plane corresponds to an eight vortex pattern much alike the secondary flow found in the turbulent regime.

\section{INTRODUCTION}

The flow through a straight duct with rectangular cross-section shares important features with both circular pipe flow and plane channel flow. Analogous to pipe flow, laminar flow in a square duct is believed to be stable with respect to all infinitesimal perturbations ${ }^{25}$. On the other hand, for aspect ratios above a critical value of $A_{\text {crit }} \approx 3.2$, duct flow is linearly unstable. In fact, ducts with large aspect ratio are often used in laboratory experiments as a substitute for plane channels ${ }^{26}$. Despite these similarities, the fact that the cross-section of rectangular ducts is neither axisymmetric nor spanwise-homogeneous leads to an interesting phenomenon: turbulent flow in this geometry exhibits mean secondary motion in the plane perpendicular to its axis ${ }^{27}$. This mean cross-flow motion is directed towards the corners in the vicinity of the diagonals, and away from the wall near the bisectors, giving rise to a pattern with eight vortices. Although of small amplitude (few percent of the primary flow), the secondary motion significantly deforms the latter, thereby inducing strong variations of local mean wall-shear stress along the edges ${ }^{28}$.

The results of recent direct numerical simulations of turbulent square duct flow suggest that the occurrence of secondary motion is a statistical footprint of the preferential location of coherent structures at certain positions along the edges ${ }^{29}$. This conclusion is based upon data obtained through a vortex eduction study applied to snapshots of turbulent flow fields at low Reynolds numbers. In contrast to these "approximate" coherent structures, "exact" coherent structures (travelling-waves, time-periodic solutions) in duct flow have only received little attention in the literature. To our knowledge, the only study presenting non-linear travelling-wave solutions for the square duct is ref. 30. However, in contrast to the claim of the authors, the solution family presented therein is not representative of the turbulent flow states observed in ref. 29. More specifically, the travelling-waves of ref. 30 exhibit a pattern of four mean secondary flow vortices which can appear in two orientations (rotated by $\pi / 2$ ). In this case what should be compared is on the one hand the statistically averaged turbulent flow field and on the other hand the average between the two (equivalent) travellingwave solutions rotated by $\pi / 2$ with respect to each other. Performing this averaging on the solution of ref. 30, however, results in a vorticity pattern with a sign which is opposite to the one of the mean secondary flow found in turbulence ${ }^{29}$.

The objective of the present work is to determine exact coherent structures which are potentially relevant to turbulent flow in a square duct. For this purpose we have computed different families of travelling-waves. In this article we present one particular solution family which entails an eight-vortex secondary flow with the same symmetries and sense of rotation as that found in timeaveraged turbulent fields. This enables us to directly compare the properties of the exact coherent structures to statistical quantities measured in turbulent flow. We are therefore in a position to give solid theoretical support to our earlier conjecture ${ }^{29,31}$ by showing that nearwall coherent structures are indeed capable of generating secondary flow in a square duct.

\section{NUMERICAL METHOD}

In the following the coordinates $\mathbf{x}=(x, y, z)$ are chosen such that $x$ is aligned with the duct axis and $y, z$ are oriented along the duct bisectors. From the duct half-width $h$, the maximum laminar flow velocity $u_{\max }$ and the kinematic viscosity $\nu$ we can form the base flow Reynolds number $R e=u_{\max } h / \nu$. We will also use the bulk flow Reynolds number $R e_{b}=u_{b} h / \nu$ (based on the bulk velocity $u_{b}$ ) as well as the friction-velocity based Reynolds number $R e_{\tau}=u_{\tau} h / \nu$ (where the wall-friction velocity $u_{\tau}$ is defined in the usual way). The superscript + will be used to represent quantities normalized with $\nu$ and $u_{\tau}$. Introducing the decomposition of the velocity field $\mathbf{u}=U(y, z) \mathbf{e}_{x}+\mathbf{u}^{\prime}(\mathbf{x}, t)$ and the pressure $p=P(x)+p^{\prime}(\mathbf{x}, t)$, the flow equations for the perturbations read:

$$
\begin{aligned}
& \partial_{t} \mathbf{u}^{\prime}+U \partial_{x} \mathbf{u}^{\prime}+\left(\mathbf{u}^{\prime} \cdot \nabla\right)\left(U \mathbf{e}_{x}+\mathbf{u}^{\prime}\right)+\nabla p^{\prime}=\nu \nabla^{2} \mathbf{u}^{\prime} \\
& \nabla \cdot \mathbf{u}^{\prime}=0, \quad \mathbf{u}^{\prime}(x, y= \pm h, z, t)=\mathbf{u}^{\prime}(x, y, z= \pm h, t)=0
\end{aligned}
$$


(a)

\begin{tabular}{lcc}
$N_{x}$ & $c_{f}$ & $\mathcal{E}$ \\
\hline 2 & 0.0361062 & $8.5279 \mathrm{e}-3$ \\
4 & 0.0364307 & $3.8270 \mathrm{e}-4$ \\
6 & 0.0364184 & $4.5376 \mathrm{e}-5$ \\
8 & 0.0364168 & $1.4451 \mathrm{e}-6$ \\
10 & 0.0364167 & $5.6946 \mathrm{e}-8$ \\
14 & 0.0364167 &
\end{tabular}

(b)

\begin{tabular}{ccc}
$N_{y}=N_{z}$ & $c_{f}$ & $\mathcal{E}$ \\
\hline 20 & 0.0320227 & 0.00526353 \\
30 & 0.0321351 & 0.00176935 \\
34 & 0.0321557 & 0.00113064 \\
40 & 0.0321759 & 0.00050299 \\
44 & 0.0321851 & 0.00021773 \\
48 & 0.0321921 &
\end{tabular}

TABLE I. Convergence of the solution with the truncation level. (a) Varying the number of Fourier modes for fixed $N_{y}=$ $N_{z}=30$ ( $R e=2345, \alpha h=1$, lower branch). (b) Varying the number of modified Chebyshev polynomials for fixed $N_{x}=2$ ( $R e=2600, \alpha h=1.5547$, lower branch). The relative error is computed with respect to the result obtained at the highest truncation level $c_{f, r e f}$, viz. $\mathcal{E} \equiv\left(c_{f}-c_{f, r e f}\right) / c_{f, r e f}$.

where $\mathbf{e}_{x}$ is the unit vector in the $x$-direction, $\mathbf{u}^{\prime}=$ $\left(u^{\prime}, v^{\prime}, w^{\prime}\right)$, the mass density has been taken as unity and $\partial_{t}=\partial / \partial t$ as well as $\partial_{x}=\partial / \partial x$. The streamwise laminar base flow component $U$ is solution of $\left(\partial^{2} / \partial y^{2}+\right.$ $\left.\partial^{2} / \partial z^{2}\right) U=R e \cdot \mathrm{d} P / \mathrm{d} x$ with $\mathrm{d} P / \mathrm{d} x=$ const. We employ a primitive variable formulation, expanding the dependent variables $\varphi=\left\{u^{\prime}, v^{\prime}, w^{\prime}, p^{\prime}\right\}$ as follows (assuming the solution to be a travelling-wave):

$$
\begin{aligned}
\varphi(\mathbf{x}, t) & =\sum_{n=-N_{x}}^{N_{x}} \sum_{m=0}^{2 N_{y}-k^{(\varphi)}} \sum_{l=0}^{2 N_{z}-k^{(\varphi)}} \varphi_{n m l} \times \\
& \times \phi_{m}^{(\varphi)}(y) \phi_{l}^{(\varphi)}(z) \exp (\operatorname{in} \alpha(x-c t)),
\end{aligned}
$$

where $\alpha$ is the streamwise wavenumber, $c$ the (realvalued) phase speed and $\mathrm{i}=\sqrt{-1}$. The functions $\phi^{(\varphi)}$ are modified Chebyshev polynomials which - in the case of $\varphi$ being a velocity component-incorporate the wall boundary conditions ${ }^{32}$. No boundary conditions are prescribed for the pressure field, and hence two polynomial degrees less than for the velocity field are used, i.e. $k^{\left(p^{\prime}\right)}=2$ and $k^{\left(u^{\prime}\right)}=k^{\left(v^{\prime}\right)}=k^{\left(w^{\prime}\right)}=0$. The equations (1) admit solutions which exhibit particular combinations of odd/even cross-sectional parities as described below. By restricting our attention to these symmetric solutions, the number of modes in the $y$ and $z$ directions can be halved without loss of resolution.

After substituting (2) into (1), a Galerkin method is employed in the streamwise (Fourier) direction, and a collocation method is used in the cross-stream (Chebyshev) directions. In our case, the collocation points for pressure are chosen as the Gauss points, i.e. pressure is staggered with respect to the usual Gauss-Lobatto grid
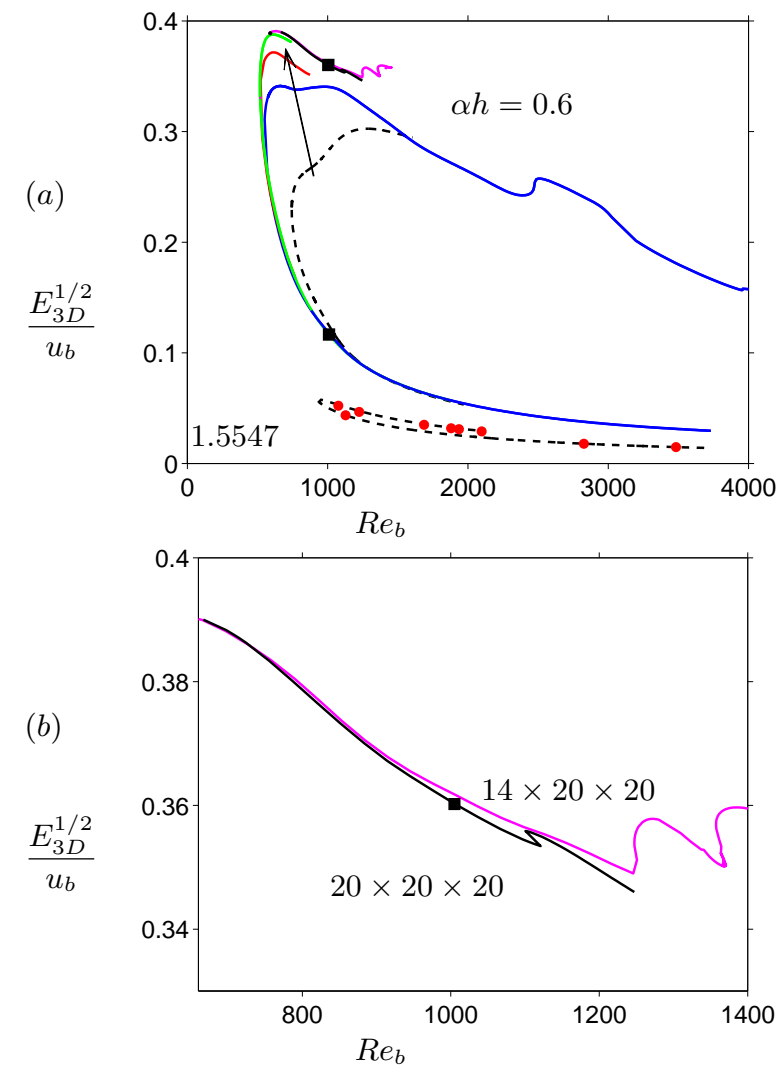

FIG. 1. Convergence of the solution curves for two different streamwise wavenumbers $\alpha h=\{0.6,1.5547\}$ computed at different truncation levels. (a) For $\alpha h=1.5547$, the dashed line corresponds to $2 \times 30 \times 30$ and the filled circles to $4 \times 32 \times 32$. For $\alpha h=0.6$, the truncation level varies along the arrow in the order: $2 \times 30 \times 30,4 \times 20 \times 20,6 \times 26 \times 26,10 \times 20 \times 20$, $14 \times 20 \times 20,20 \times 20 \times 20$, with the filled squares indicating individual solutions at $20 \times 26 \times 26$. (b) Close-up of the data shown in $(a)$ for $\alpha h=0.6$ on the upper branch. The curve for the truncation level $20 \times 20 \times 20$ is drawn up to the point where the relative difference with respect to the solution obtained at $14 \times 20 \times 20$ is still less than one percent.

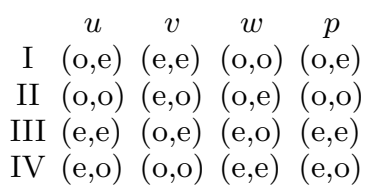

TABLE II. The four combinations of cross-sectional solution parities admitted by the linearized operator, as shown in ref. 25. The notation is such that e.g. "(o,e)" stands for an odd parity in $y$ and an even parity in $z$. 


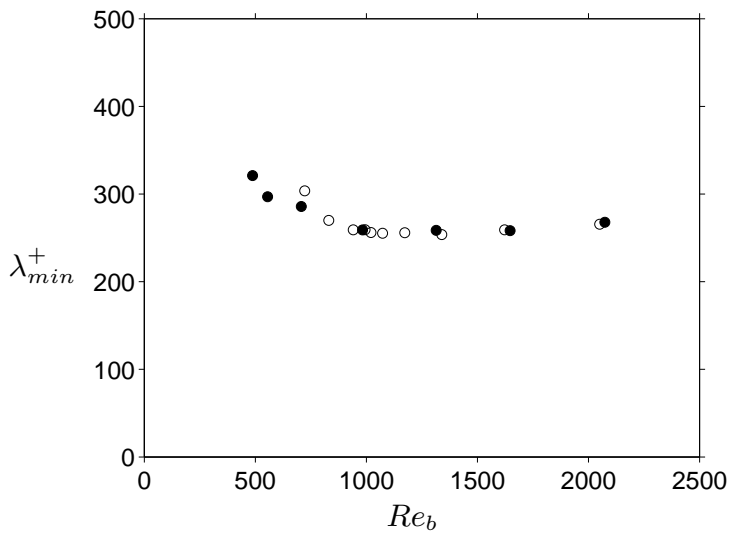

FIG. 2. Minimum wavelength (in wall units) of the travellingwaves given as a function of the bulk Reynolds number. The open symbols correspond to a truncation level of $2 \times 30 \times 30$, the filled ones to $4 \times 20 \times 20$.

used for velocity, thereby avoiding a discretization of the continuity equation at the corner points. It should be noted that the above discretization of the space operator guarantees a solution which is free from spurious pressure modes ${ }^{33}$. The solution of the resulting nonlinear algebraic system is performed via Newton-Raphson iteration, the linear system being solved by means of LU decomposition, exploiting parallel computing techniques. Continuation along the different problem parameters is achieved through an arc-length procedure ${ }^{34}$. It has been verified that our code reproduces the solution of ref. 30 with high accuracy (choosing $\alpha h=0.85$ leads to $\min \left(R e_{b}\right)=596.1191$ and $c / u_{\max }=0.4780$ when using $N_{x}=4, N_{y}=N_{z}=26$ ). Furthermore, numerical convergence of the solutions presented in the following has been thoroughly investigated. Table I shows the

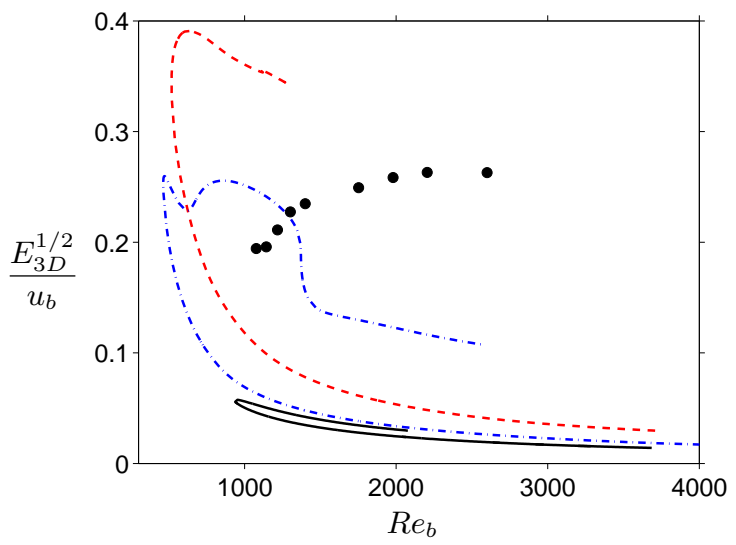

FIG. 3. Three-dimensional perturbation velocity, normalized by the bulk velocity, as a function of the bulk Reynolds number. The lines connect solutions at constant streamwise wavenumber: $\alpha h=0.6$ (dashed), $\alpha h=1$ (chain-dotted), $\alpha h=1.5547$ (solid). The symbols $(\bullet)$ are for turbulent flow ${ }^{29}$.

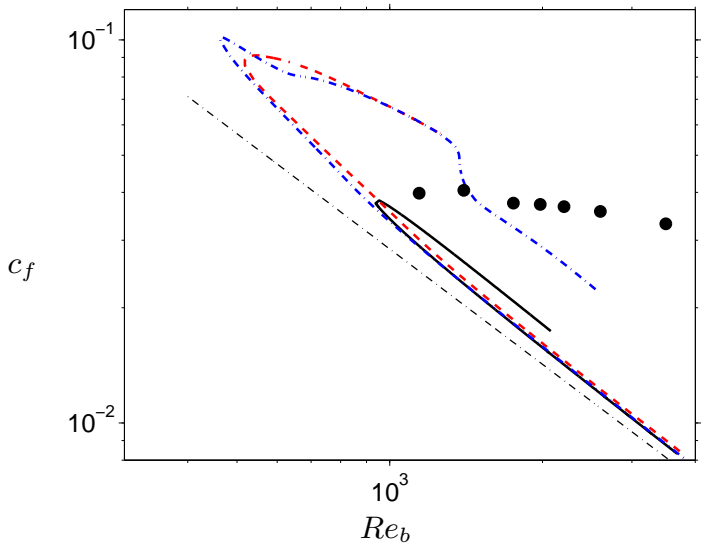

FIG. 4. Wall friction factor $c_{f}=8 u_{\tau}^{2} / u_{b}^{2}$ as a function of $R e_{b}$ in logarithmic scale ( $u_{\tau}$ being the friction velocity). Line styles and symbols as defined in figure 3 . The additional straight chain-dotted line indicates laminar friction.

convergence with respect to the number of streamwise Fourier modes as well as the cross-stream polynomials for two particular parameter points. It was found that the truncation level required for resolving the present solution family varies considerably in parameter space. In particular, resolving the upper branch solution at small wavenumbers imposes the most stringent requirements (as previously observed in Couette flow ${ }^{35}$ ). This point is illustrated in figure 1 , where solution curves at two different streamwise wavenumbers are depicted as computed with different truncation levels. The plotted quantity is the perturbation energy which excludes the streamwiseconstant modes, i.e. $E_{3 D}=\sum_{n \neq 0} \iint\left|\hat{\mathbf{u}}_{n}\right|^{2} \mathrm{~d} y \mathrm{~d} z$ where $\hat{\mathbf{u}}_{n}$ is the $n$th Fourier coefficient of velocity. The figure shows that $N_{x}=2$ suffices for $\alpha h=1.5547$, while the smaller wavenumber value $\alpha h=0.6$ requires a considerably higher number of streamwise modes. In particular, we have used a streamwise truncation level of up to $N_{x}=20$ on the upper branch for $\alpha h=0.6$. With a resolution of $N_{x}=20$ and $N_{y}=N_{z}=20$ we obtain results which are within one percent of the results obtained for $N_{x}=14$ and $N_{y}=N_{z}=20$ up to $R e=6565$ (corresponding to $R_{b}=1247.2$ ). Beyond that point, results obtained with these two truncation levels exhibit substantially larger differences, and our finest solution will therefore only be discussed up to $R_{b}=1247.2$ in the following. These tough resolution requirements stem from the fact that the flow field on the upper branch at smaller wavenumbers develops very strong streamwise gradients as can be seen in figure 5 below.

In the absence of a "natural" primary bifurcation point from which a reasonable initial guess can be constructed, we resort to the homotopy method proposed by Waleffe ${ }^{36}$ based on the so-called self-sustaining process of wallbounded shear flows. Here streamwise vortices are artificially added to the base flow and forced against viscous decay, leading to streaks, which are in turn linearly 
unstable, feeding back into the original vortices. The non-linear solution is then continued back to the original problem, i.e. the unforced flow. As suggested by Waleffe $^{36}$ we select the streamwise rolls amongst the least decaying eigenmodes of the Stokes operator in the crosssectional plane. In the following we will focus upon results obtained using the fourth eigenmode ${ }^{37}$ which exhibits a parity of type III in the nomenclature of ref. 25 (cf. table II). In fact, the non-linear equations admit solutions which are such that all even streamwise Fourier modes verify the parity type III and all odd Fourier modes simultaneously verify one of the parity types I, II, III or IV. This property allows us to reduce the system size while choosing any linear streak instability mode in conjunction with rolls of parity III. The following results were obtained when starting at a primary bifurcation point of a linear perturbation with parity II.

\section{RESULTS}

The critical point (minimum value of the bulk Reynolds number) is found at $\min \left(R e_{b}\right)=471$ for $\alpha h=0.927$ (determined at a truncation level of $N_{x}=4$, $N_{y}=N_{z}=26$ ); this corresponds to a value of the friction-velocity based Reynolds number of $R e_{\tau}=51.6$. It should be recalled that self-sustained turbulence in square duct flow exists above $R e_{b} \approx 1070^{29}\left(R e_{\tau} \approx 80\right)$; localized turbulent "puffs" have been found to subsist above $R e_{b} \approx 800$ (unpublished results). The region in parameter space which is occupied by this travelling-wave family has an upper bound in wavenumber $\alpha$ which increases with the Reynolds number; when normalized in wall units the corresponding short wavelength limit $\lambda_{\min }^{+}$ is relatively constant with a value between 250 and 300 over a considerable range of $R e_{b}$ (figure 2). This shortest streamwise wavelength at which the travelling-waves appear is not very far from the length of the minimal flow unit for self-sustained turbulence which measures roughly 190 wall units in the square duct ${ }^{29}$ and 250-300 in plane channel flow ${ }^{38}$. It should be noted that a precise determination of the upper limit of the streamwise wavelength as function of Reynolds number is beyond the scope of the present paper.

Figure 3 characterizes the solution in terms of the three-dimensional perturbation energy. Here and in the following we will focus upon three specific values of the streamwise wavenumber, namely $\alpha h=\{0.6,1.0,1.5547\}$. It can be seen that the quantity $E_{3 D}^{1 / 2} / u_{b}$ varies significantly with the streamwise wavenumber (especially on the upper branch), the highest values being observed for the smallest of the three wavenumbers $(\alpha h=0.6)$. The figure also includes the time-averaged counterpart of the three-dimensional perturbation energy for turbulent flow (obtained through $\mathrm{DNS}^{29}$ ). At low Reynolds number the present solutions with wavenumber $\alpha h=\{0.6,1\}$ are more energetic than the average turbulent flow, while the opposite is true for $\alpha h=1$ at higher Reynolds number
$R e_{b} \gtrsim 1370 ;$ unfortunately, due to the above mentioned high resolution requirements, it was not possible to further continue the curve for $\alpha h=0.6$ in order to judge whether a similar drop-off occurs at that wavenumber.

Let us now turn to the friction factor. Figure 4 demonstrates that the lower branch of the present solution surface scales approximately as the laminar solution (i.e. varying as $R e_{b}^{-1}$ ) at all wavenumbers. The upper branch exhibits significantly higher friction values at smaller wavenumbers. On the upper branch curves corresponding to $\alpha h=0.6$ and $\alpha h=1$ the average turbulent friction values are exceeded for small Reynolds numbers; again, cross-over between the travelling-waves at $\alpha h=1$ and the turbulent data takes place at $R e_{b} \approx 1370$, beyond which the upper branch solution scales approximately as $R e_{b}^{-1}$. Conversely, for the larger wavenumber $(\alpha h=1.5547)$ the upper branch has a similar slope to the lower branch at all Reynolds numbers and does not correspond to significantly increased friction with respect to the laminar base flow.

In figure 5 solutions on the upper and lower branch at the same wavelength $(\alpha h=1)$ and similar bulk Reynolds number $\left(R e_{b} \approx 1400\right)$ are depicted by means of isosurfaces of streamwise vorticity $\omega_{x}$ and total streamwise velocity $u$. The former isosurfaces take the shape of streamwise elongated tubes which are slightly inclined with respect to the wall plane. Surfaces of the streamwise velocity exhibit a single corrugation per wall, which corresponds to a low-speed streak. The streamwise vortices are in streamwise alternating (staggered) arrangement with respect to the nearest streak. When focusing upon structures in the vicinity of a single wall, it becomes clear that the present travelling-waves are similar to exact coherent structures found in plane wall-bounded shear flows ${ }^{36}$. Furthermore, the staggered vortex/lowspeed streak configuration described above is at the center of a multitude of observations in the near-wall region of turbulent flow (e.g. DNS of Jeong et al. ${ }^{39}$ ) as well as in time-periodic solutions ${ }^{40}$.

The lower branch solution in figure 5 exhibits a very mild streamwise dependency (i.e. the streaks are nearly straight), as well as very smoothly shaped, large-scale streamwise vortices. This is consistent with the fact that the shape of the lower branch solution changes very little with Reynolds number. The upper branch, on the other hand, is characterized by very localized vortices and relatively sharp bending of the streaks. The high intensity of the vortices in the upper branch solution as well as their proximity to the walls is manifested through the existence of patches with strong mirror vorticity.

Figure 5 also shows the streamwise-averaged flow corresponding to the upper/lower branch solutions. A secondary flow pattern with eight vortices, very much alike the one exhibited on average by turbulent duct flow, can be observed. The resulting deformation of the primary flow contours leads to a variation of wall-shear along the edges (not shown), which is characterized by two local maxima near the corners and a local minimum at the bi- 

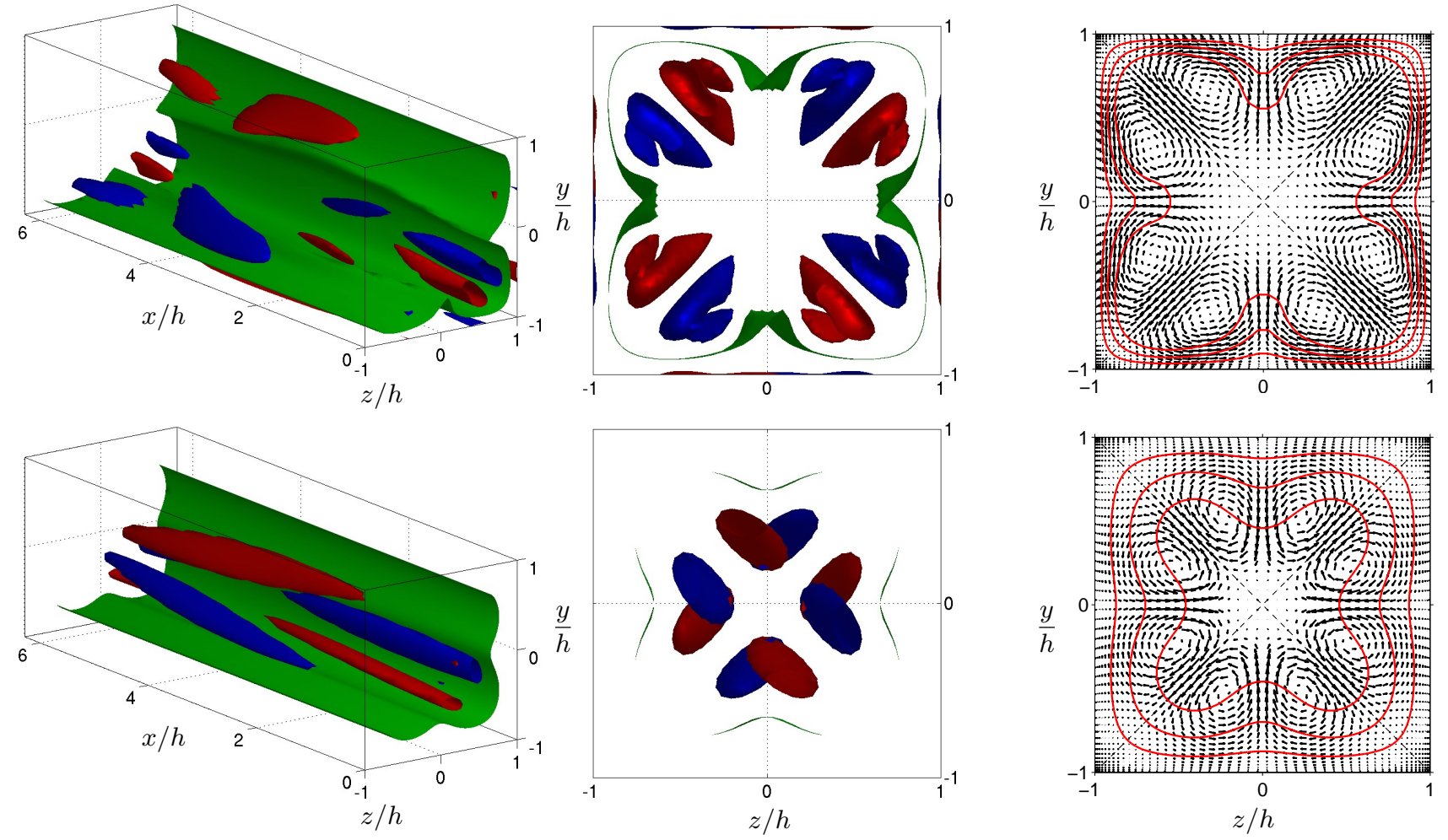

FIG. 5. Top row: the upper branch solution at $R e_{b}=1404.1$; bottom row: the lower branch solution at $R e_{b}=1398.3$; both at $\alpha h=1$. The graphs in the left and center column show surfaces of constant values of the total streamwise velocity $u=0.55 \max (u)$ (sheet-like structure near the walls, green color) and of the streamwise vorticity at $\pm 0.65( \pm 0.55)$ times its maximum absolute value in the case of the upper (lower) branch solution (tubular structures, blue/red color). This value corresponds to $\omega_{x}^{+}= \pm 0.0655\left(\omega_{x}^{+}= \pm 0.040\right)$. In the left column the surfaces are only shown on one side of a diagonal of the duct cross-section. The right column depicts the corresponding mean flow, the primary flow $\langle u\rangle$ being visualized by contourlines at $\{0.25,0.5,0.75\}$ times the maximum value, and the secondary flow $(\langle v\rangle,\langle w\rangle)$ in form of arrows. Truncation level: $N_{x}=20$, $N_{y}=N_{z}=26$. 
sector - as found in turbulent flow ${ }^{31}$ at Reynolds numbers below $R e_{b} \approx 2000$. Figure 6 shows the statistically averaged mean flow from $\mathrm{DNS}^{31}$ at $R e_{b}=1400$, facilitating a direct comparison with the present results of figure 5 at practically the same Reynolds number. A strong similarity of primary flow contours and secondary flow pattern between DNS data and the upper branch travelling-wave can indeed be observed.

In order to quantify the amplitude of the streamwise averaged secondary motion the following energy norm can be defined: $E_{v w}=\iint\left(\left|\hat{v}_{0}\right|^{2}+\left|\hat{w}_{0}\right|^{2}\right) \mathrm{d} y \mathrm{~d} z$. The square-root of this quantity, normalized by the bulk velocity, is shown in figure 7. Analogously to the perturbation energy (cf. figure 3), the present travelling-waves (at small wavenumbers) by far surpass the average secondary flow intensity of turbulence at lower Reynolds numbers. Again a steep drop occurs for $\alpha h=1$ around $R e_{b} \approx 1370$, after which the travelling-waves and turbulence exhibit secondary flow of similar magnitude (to within $50 \%$ of each other). While the turbulent data for $E_{v w}^{1 / 2} / u_{b}$ appears to approach a constant value at higher Reynolds numbers, no scaling of the present upper-branch solutions can be deduced from our data. On the other hand, the curves for the lower branch practically collapse at all wavenumbers and vary approximately as $E_{v w}^{1 / 2} / u_{b} \sim R e_{b}^{-1}$, implying a scaling with the viscous velocity, i.e. $E_{v w}^{1 / 2} \sim \nu / h$; this scaling has previously been observed in Couette flow ${ }^{41}$ and pipe flow ${ }^{42}$.

The phase velocity (shown in figure 8) corresponding to the present lower-branch solutions tends towards values in the range of $c / u_{b}=1.66-1.75$ (depending on the wavenumber), which is comparable to the asymptotic value 1.76 of the lower-branch solution with mirror symmetry in pipe flow ${ }^{42}$. On the upper branch and for small wavenumbers, the values for the phase speed of the present solutions are in the range of $c / u_{b}=1.2-1.4$. In terms of the friction velocity, the lowest phase-speed $c_{\text {min }}^{+} \approx 12$ is obtained near the turning point, i.e. the junction of lower and upper branches (for both wavenumbers 0.6 and 1 ). This value is comparable to the propagation speed of near-wall coherent structures in turbulent channel flow, which is equal to approximately 10 times the friction velocity ${ }^{43}$.

\section{CONCLUSION}

The present non-linear equilibrium solutions to the Navier-Stokes equations are characterized by coherent structures (staggered streamwise vortices and wavy streaks) commonly found in wall-bounded shear flows. Their streamwise average also corresponds to the pattern of the time-averaged velocity field observed in (laboratory and numerical) experiments of turbulent square duct flow. Therefore, they demonstrate (for the first time theoretically) that near-wall coherent structures are indeed capable of generating physically correct secondary motion in duct flow (as previously conjectured ${ }^{29,31}$ ). Furthermore, we have shown that the present family of travelling-waves yields integral flow quantities which are generally comparable to statistical data of turbulent flow. This implies that these equilibrium solutions are potentially relevant to the turbulent state. In particular, it was shown that the upper branch solution at an intermediate streamwise wavenumber $(\alpha h=1)$ yields secondary flow intensities which are comparable to turbulence data for $R e_{b} \geq 1370$; furthermore, the upper-branch solution changes from a highly energetic and high wall friction state at lower Reynolds numbers to a low-intensity state for higher $R e_{b}$, crossing the statistical energy and wall friction level of turbulence around $R e_{b} \approx 1370$. Interestingly, this drop in perturbation energy and wall friction coincides with the Reynolds number range where turbulent duct flow exhibits a transition in terms of the instantaneous flow pattern: below $R e_{b} \approx 1250$ the predominant state of turbulent flow is characterized by a vortex pair and a single low-speed streak adjacent to one pair of opposite walls (the so-called 4-vortex state), whereas this feature gradually disappears with increasing Reynolds number, giving way to an 8-vortex state (vortex pair/streak present near all four walls simultaneously $)^{29}$. Therefore, it can be expected that the present solution family is pertinent to turbulence-induced mean secondary motion in square ducts at $R e_{b} \gtrsim 1370$. Furthermore, DNS shows that the average number of streaks which are simultaneously present along one edge of the cross-section increases with the Reynolds number ${ }^{31}$, consistent with a scaling of the spanwise streak-spacing in wall units. This property implies that additional travelling-waves with a distinct vortex pattern $(16,24$, $32, \ldots$ vortices in total, i.e. $5,7,9, \ldots$ streaks per edge) are expected to come into play progressively as the Reynolds number is increased. As a consequence, we expect our present family of exact coherent structures to be consistent with actual buffer-layer structures in turbulent flow only around $R e_{b} \approx 1370$.

It should be mentioned that other families of travellingwaves which are also consistent with the coherent bufferlayer structures and the average secondary flow pattern encountered in square duct turbulence might very well exist. However, the central point of the present communication is the fact that such a non-linear solution does indeed exist at all.

In order to determine which role the present equilibrium solutions play in the dynamics of the initial value problem, the stability characteristics of the travellingwaves should first be investigated. Then actual turbulent phase-space trajectories need to be mapped out, and their relation to the travelling waves (fixed points) needs to be established (as e.g. performed for Couette flow in ref. 44). This analysis will be the object of a future study. 


\section{ACKNOWLEDGMENTS}

The authors are grateful to A. Sekimoto for providing the DNS data visualized in figure 6 .

1

20 .

${ }^{3}$ T. Tatsumi and T. Yoshimura. Stability of the laminar flow in a rectangular duct. J. Fluid Mech., 212:437-449, 1990.

${ }^{4}$ M. Nishioka and M. Asai. Some observations of the subcritical transition in plane Poiseuille flow. J. Fluid Mech., 150:441-450, 1985.

${ }^{5}$ J. Nikuradse. Untersuchung über die Geschwindigkeitsverteilung in turbulenten Strömungen. VDI Forschungsarbeiten auf dem Gebiete des Ingenieurwesens, 281:1-44, 1926

${ }^{6}$ S. Gavrilakis. Numerical simulation of low-Reynolds-number turbulent flow through a straight square duct. J. Fluid Mech., 244:101-129, 1992.

${ }^{7}$ M. Uhlmann, A. Pinelli, G. Kawahara, and A. Sekimoto. Marginally turbulent flow in a square duct. J. Fluid Mech. 588:153-162, 2007

${ }^{8}$ H. Wedin, A. Bottaro, and M. Nagata. Three-dimensional travelling waves in a square duct. Phys. Rev. E, 79:065305(R), 2009.

${ }^{9}$ A. Pinelli, M. Uhlmann, A. Sekimoto, and G. Kawahara. Reynolds number dependence of mean flow structure in square duct turbulence. J. Fluid Mech., 644:107-122, 2010.

${ }^{10} \mathrm{M}$. Uhlmann and M. Nagata. Linear stability analysis of flow in an internally heated rectangular duct. J. Fluid Mech., 551:387404, 2006

${ }^{11}$ C. Canuto, M.Y. Hussaini, A. Quarteroni, and T.A. Zang. Spectral methods. Evolution to complex geometries and applications to fluid dynamics. Springer, 2007.

${ }^{12} \mathrm{~W}$. Rheinboldt and J. Burkardt. A locally parameterized continuation process. ACM Transactions on Mathematical Software, 9(2):215-235, 1983.

${ }^{13}$ J. Jiménez, G. Kawahara, M.P. Simens, M. Nagata, and M. Shiba. Characterization of near-wall turbulence in terms of equilibrium and "bursting" solutions. Phys. Fluids, 17:015105, 2005

${ }^{14} \mathrm{~F}$. Waleffe. Homotopy of exact coherent structures in plane shear flows. Phys. Fluids, 15(6):1517-1534, 2003.

${ }^{15}$ E. Leriche and G. Labrosse. Stokes eigenmodes in square domain and the streamfunction-vorticity correlation. J. Comput. Phys, 200:489-511, 2004.

${ }^{16} \mathrm{~J}$. Jiménez and P. Moin. The minimal flow unit in near-wall turbulence. J. Fluid Mech., 225:213-240, 1991.

${ }^{17}$ J. Jeong, F. Hussain, W. Schoppa, and J. Kim. Coherent structures near the wall in a turbulent channel flow. J. Fluid Mech., 332:185-214, 1997.

${ }^{18}$ G. Kawahara and S. Kida. Periodic motion embedded in plane Couette turbulence: regeneration cycle and burst. J. Fluid Mech., 49:291-300, 2001.

${ }^{19}$ J. Wang, J. Gibson, and F. Waleffe. Lower branch coherent states in shear flows: transition and control. Phys. Rev. Lett., 98:204501, 2007.

${ }^{20} \mathrm{D}$. Viswanath. The critical layer in pipe flow at high Reynolds number. Proc. Roy. Soc. A, 367:561-576, 2008.

${ }^{21} \mathrm{~J}$. Kim and F. Hussain. Propagation velocity of perturbations in turbulent channel flow. Phys. Fluids A, 5(3):695-706, 1993.

${ }^{22}$ J.F. Gibson, J. Halcrow, and P. Cvitanović. Visualizing the dynamics of state space in Couette flow. J. Fluid Mech., 611:107$130,2008$.

${ }^{24} 0$.

${ }^{25}$ T. Tatsumi and T. Yoshimura. Stability of the laminar flow in a rectangular duct. J. Fluid Mech., 212:437-449, 1990.

${ }^{26}$ M. Nishioka and M. Asai. Some observations of the subcritical transition in plane Poiseuille flow. J. Fluid Mech., 150:441-450, 1985.

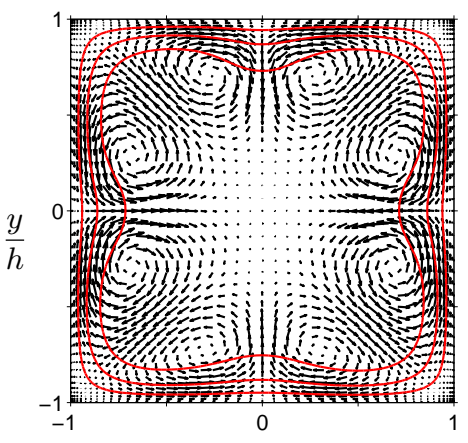

${ }^{27}$ J. Nikuradse. Untersuchung über die Geschwindigkeitsverteilung in turbulenten Strömungen. VDI Forschungsarbeiten auf dem Gebiete des Ingenieurwesens, 281:1-44, 1926.

${ }^{28}$ S. Gavrilakis. Numerical simulation of low-Reynolds-number turbulent flow through a straight square duct. J. Fluid Mech., 244:101-129, 1992.

${ }^{29}$ M. Uhlmann, A. Pinelli, G. Kawahara, and A. Sekimoto. Marginally turbulent flow in a square duct. J. Fluid Mech., 588:153-162, 2007

${ }^{30}$ H. Wedin, A. Bottaro, and M. Nagata. Three-dimensional travelling waves in a square duct. Phys. Rev. E, 79:065305(R), 2009

${ }^{31}$ A. Pinelli, M. Uhlmann, A. Sekimoto, and G. Kawahara. Reynolds number dependence of mean flow structure in square duct turbulence. J. Fluid Mech., 644:107-122, 2010.

${ }^{32} \mathrm{M}$. Uhlmann and M. Nagata. Linear stability analysis of flow in an internally heated rectangular duct. J. Fluid Mech., 551:387404, 2006.

${ }^{33}$ C. Canuto, M.Y. Hussaini, A. Quarteroni, and T.A. Zang. Spectral methods. Evolution to complex geometries and applications to fluid dynamics. Springer, 2007.

${ }^{34} \mathrm{~W}$. Rheinboldt and J. Burkardt. A locally parameterized continuation process. ACM Transactions on Mathematical Software, $9(2): 215-235,1983$.

${ }^{35}$ J. Jiménez, G. Kawahara, M.P. Simens, M. Nagata, and M. Shiba. Characterization of near-wall turbulence in terms of equilibrium and "bursting" solutions. Phys. Fluids, 17:015105, 2005.

${ }^{36} \mathrm{~F}$. Waleffe. Homotopy of exact coherent structures in plane shear flows. Phys. Fluids, 15(6):1517-1534, 2003.

${ }^{37}$ E. Leriche and G. Labrosse. Stokes eigenmodes in square domain and the streamfunction-vorticity correlation. J. Comput. Phys, 200:489-511, 2004.

${ }^{38}$ J. Jiménez and P. Moin. The minimal flow unit in near-wall turbulence. J. Fluid Mech., 225:213-240, 1991.

${ }^{39}$ J. Jeong, F. Hussain, W. Schoppa, and J. Kim. Coherent structures near the wall in a turbulent channel flow. J. Fluid Mech., 332:185-214, 1997.

${ }^{40}$ G. Kawahara and S. Kida. Periodic motion embedded in plane Couette turbulence: regeneration cycle and burst. J. Fluid Mech., 49:291-300, 2001.

${ }^{41}$ J. Wang, J. Gibson, and F. Waleffe. Lower branch coherent states in shear flows: transition and control. Phys. Rev. Lett., 98:204501, 2007.

${ }^{42} \mathrm{D}$. Viswanath. The critical layer in pipe flow at high Reynolds number. Proc. Roy. Soc. A, 367:561-576, 2008.

${ }^{43} \mathrm{~J}$. Kim and F. Hussain. Propagation velocity of perturbations in turbulent channel flow. Phys. Fluids A, 5(3):695-706, 1993.

${ }^{44}$ J.F. Gibson, J. Halcrow, and P. Cvitanović. Visualizing the dynamics of state space in Couette flow. J. Fluid Mech., 611:107130, 2008. 


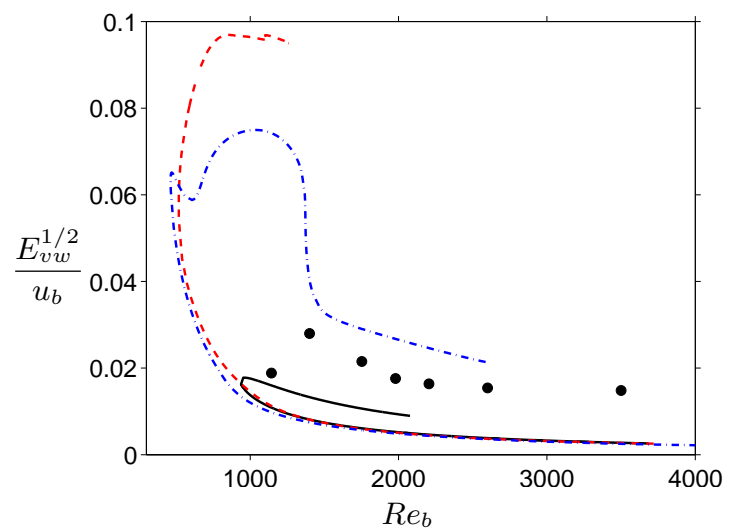

FIG. 7. The secondary flow intensity of the present solution family, normalized by the bulk velocity and shown as a function of $R e_{b}$. Line styles and symbols as defined in figure 3 .

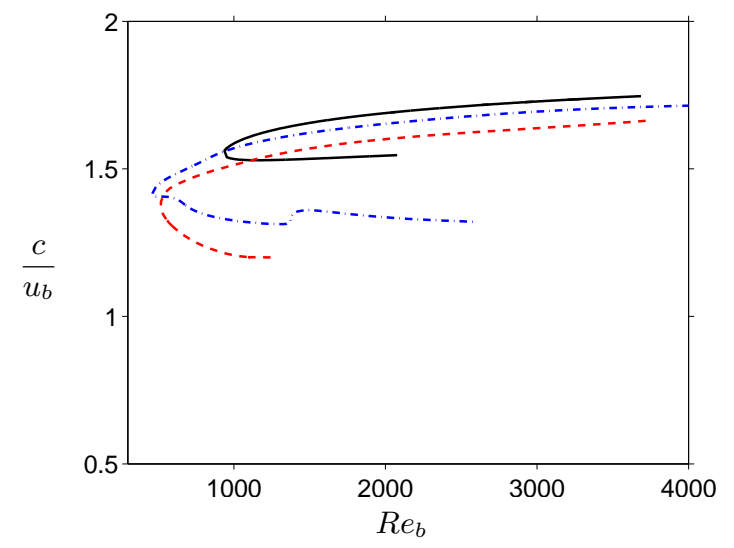

FIG. 8. The phase velocity normalized by the bulk velocity. Line styles as defined in figure 3 . 